Supplementary material

\title{
Contrastive Study of In-Situ Sensing and Swabbing Detection Based on SERS-Active Gold Nanobushes-PDMS Hybrid Film
}

\author{
Yi Ma, Yiran Tian, Ying Chen, Chenjie Gu, Tao Jiang* \\ ${ }^{a}$ Department of Microelectronic Science and Engineering, School of Physical Science and \\ Technology, Ningbo University, Ningbo 315211, Zhejiang, P. R. China
}

*Corresponding author: Tao Jiang, E-mail: jiangtao@nbu.edu.cn, 818 Fenghua Road, Ningbo 315211 China 

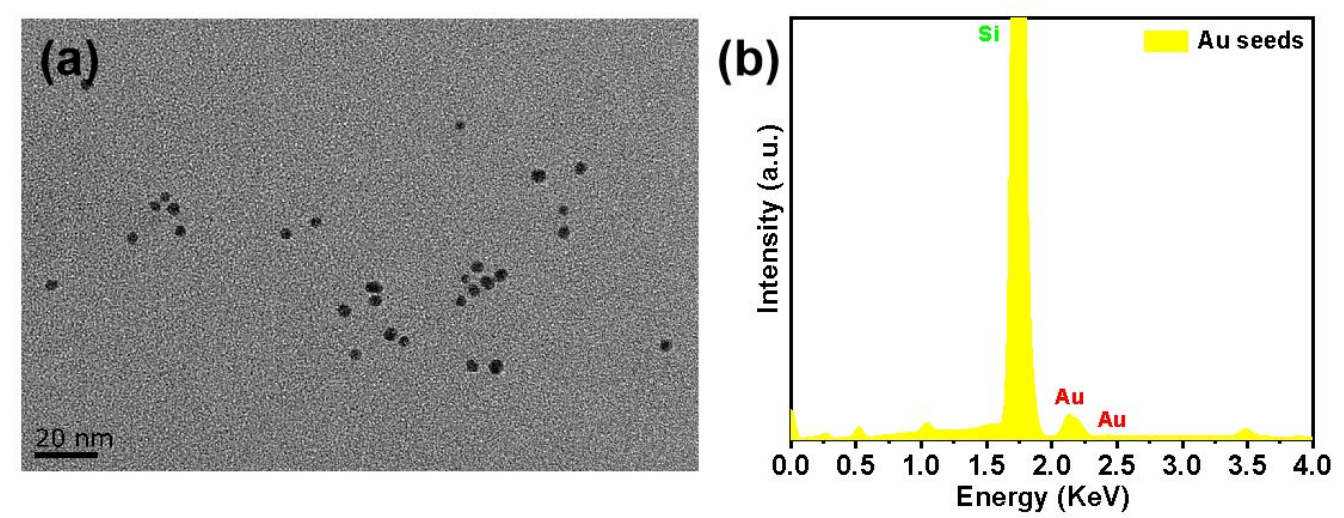

Figure S1. (a) TEM image and (b) EDS spectrum of Au seeds. 

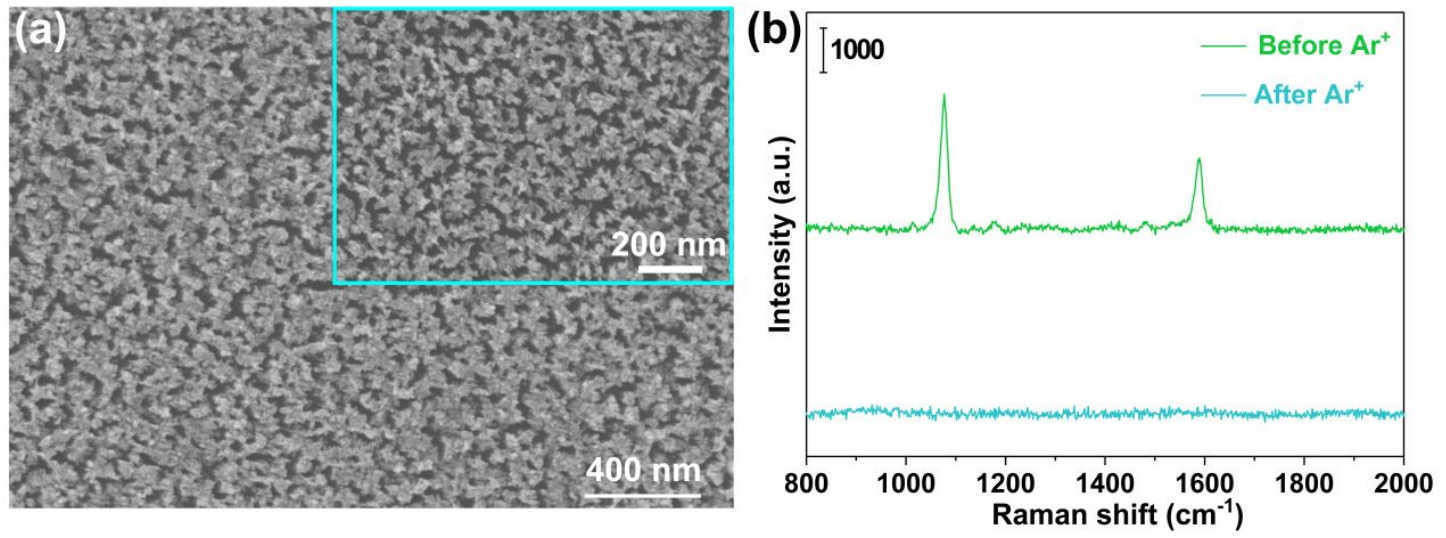

Figure S2. (a) SEM image of $\mathrm{Ar}^{+}$plasma treated $\mathrm{Au}$ NBs synthesized with $0.25 \mathrm{mM}$ of 4MBA; (b) Raman spectra of Au NBs on PDMS film before and after $\mathrm{Ar}^{+}$plasma treatment. 


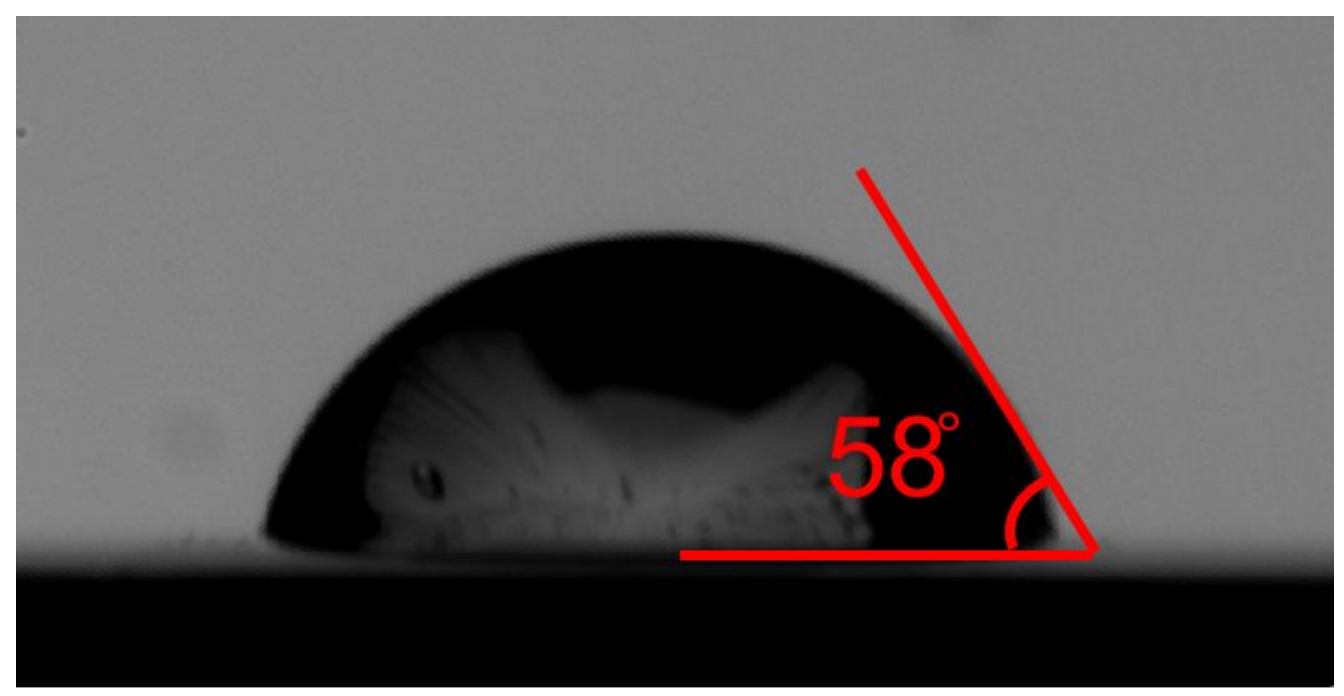

Figure S3. Water contact angle of the Au NBs-PDMS hybrid film. 


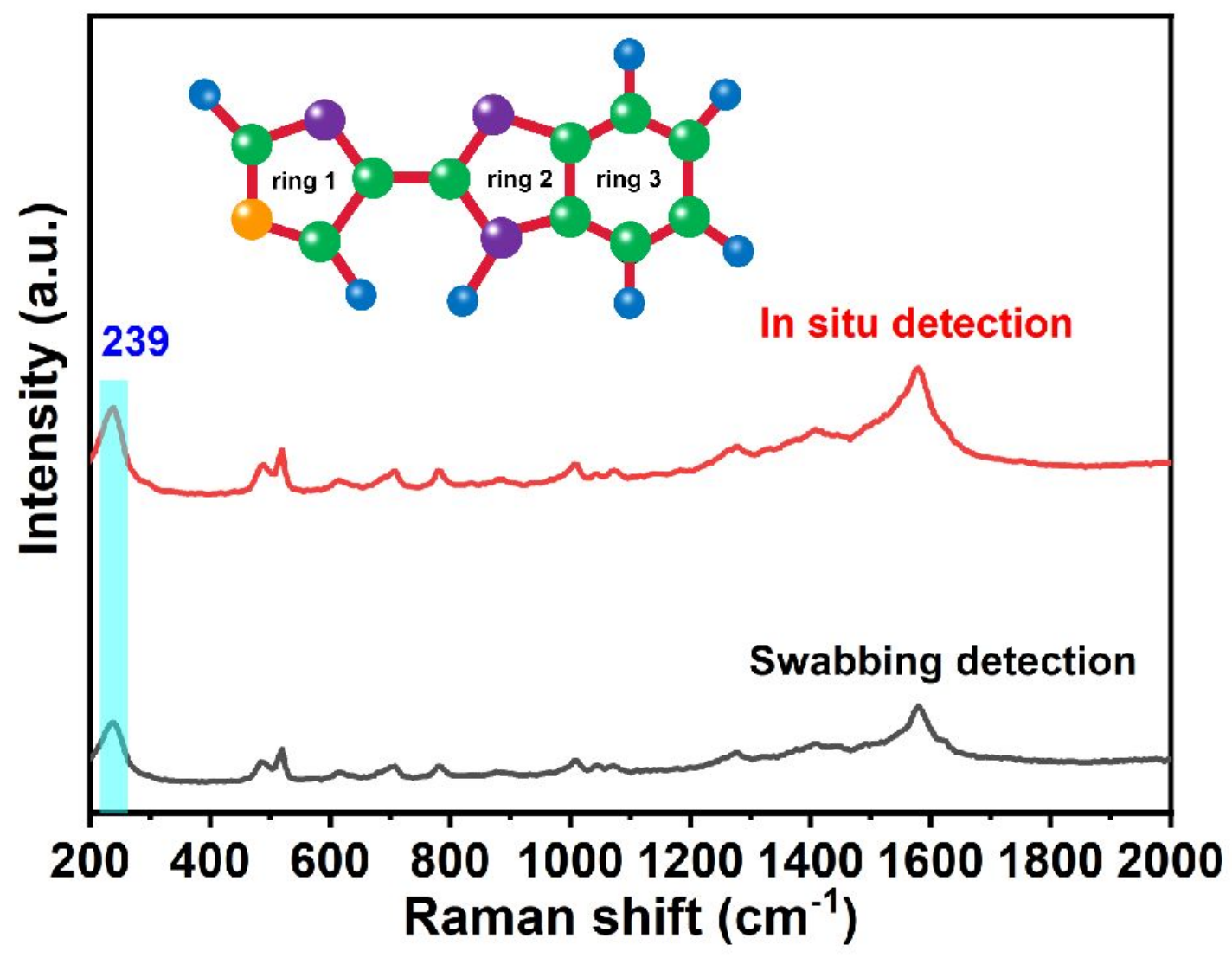

Figure S4. SERS peaks of TBZ at $239 \mathrm{~cm}^{-1}$ obtained by in-situ and swabbing detection. 